\title{
Biomarkers and immunoprofiles associated with fetal abnormalities of ZIKV-positive pregnancies
}

\author{
Suan-Sin Foo, ${ }^{1}$ Weiqiang Chen, ${ }^{1}$ Yen Chan, ${ }^{2}$ Wai-Suet Lee, ${ }^{1,3}$ Shin-Ae Lee, ${ }^{1}$ Cenhong Cheng, ${ }^{4}$ Karin \\ Nielsen-Saines, ${ }^{5}$ Patrícia Brasil, ${ }^{6}$ and Jae U. Jung ${ }^{1}$ \\ 'Department of Molecular Microbiology and Immunology and '2Division of Maternal-Fetal Medicine, Department of \\ Obstetrics and Gynecology, Keck School of Medicine, University of Southern California, Zilkha Neurogenetic Institute, \\ Los Angeles, California, USA. Institute for Glycomics, Griffith University, Gold Coast, Southport, Queensland, Australia. \\ ${ }^{4}$ Department of Microbiology and Molecular Genetics and ${ }^{5}$ Division of Pediatric Infectious Diseases, David Geffen School of \\ Medicine, UCLA, Marion Davies Children's Health Center, Los Angeles, California, USA. 'Laboratório de Pesquisa Clínica em \\ Doenças Febris Agudas, Instituto Nacional de Infectologia Evandro Chagas, Fondação Oswaldo Cruz (FIOCRUZ), \\ Rio de Janeiro, Brazil.
}

BACKCROUND. An intricate fetal-maternal immune crosstalk during pregnancy is essential for a healthy birth. Hence, the infection-induced alterations of maternal immunity often lead to adverse outcomes for mother and/or child. The emergence of Zika virus (ZIKV) infection in pregnant women has been associated with more than 3,000 cases of microcephaly and nervous system malformations.

METHODS. To explore the potential correlation of ZIKV-induced alteration of maternal immunity with fetal abnormalities, we performed extensive sera immunoprofiling of $\mathbf{7 4}$ pregnant women: 30 symptomatic ZIKV+ pregnant patients and 30 healthy pregnant controls in ZIKV-endemic Rio de Janeiro, along with 14 healthy pregnant controls in non-endemic Los Angeles.

RESULTS. Extensive multiplexing analysis of 69 cytokines revealed that CXCL10, CCL2, and CCL8 chemokines were specifically associated with symptomatic ZIKV+ infection during pregnancy, and distinct immunoprofiles were detected at different trimesters in ZIKV-infected pregnant women. Intriguingly, the high CCL2 level and its inverse correlation with CD163, TNFRSF1A, and CCL22 levels was apparently associated with ZIKV-induced abnormal birth.

CONCLUSION. Our findings provide insights into the alteration of ZIKV-elicited maternal immunity, serving as a potential clinical biomarker platform.

FUNDING. NIH (CA200422, CA180779, DE023926, Al073099, Al116585, Al129496, Al140705, Al069120, Al056154, Al078389, Al28697, Al40718 and Al129534-01), Hastings Foundation, Fletcher Jones Foundation, Departamento de Ciência e Tecnologia (DECIT/25000.072811/2016-17) do Ministério da Saúde do Brasil, and Coordenação de Aperfeiçoamento de Pessoal de Nivel Superior CAPES/88887.116627/2016-01.

Authorship note: SSF and WC contributed equally to this work.

Conflict of interest: The authors have declared that no conflict of interest exists.

License: Copyright 2018, American Society for Clinical Investigation.

Submitted: August 9, 2018 Accepted: September 19, 2018 Published: November 2, 2018

Reference information: JCI Insight. 2018;3(21):e124152. https://doi.org/10.1172/jci. insight.124152.

\section{Introduction}

Since the first reported cases of Zika virus-related (ZIKV-related) microcephaly in late 2015, new clinical and experimental evidence has emerged to support ZIKV as the viral etiology (1). To date, approximately 800,000 cases of suspected and confirmed autochthonous ZIKV infections have been reported in the Americas (2). According to data from the Centers for Disease Control and Prevention, approximately 5,000 pregnant women have been infected with ZIKV in the United States. Among those, there were more than 200 cases ( $\sim 5 \%)$ of confirmed fetal defects and close to 20 cases $(\sim 0.5 \%)$ of pregnancy loss (3). Despite sporadic outbreaks in various countries, the majority of ZIKV-associated birth defects were reported in Brazil which accounted for almost 400,000 cases of infections and approximately 3,000 cases of confirmed congenital defects. In US territories, $5 \%$ of the fetuses or infants born to $\mathrm{ZIKV}$-positive $\left(\mathrm{ZIKV}^{+}\right)$women were reported 
to have possible virus-associated birth defects $(4,5)$. We utilized blood specimens from a prospective cohort of pregnant women in Rio de Janeiro, Brazil, in which an alarmingly high rate (up to 42\%) of babies born to $\mathrm{ZIKV}^{+}$mothers were reported to have birth defects (6). According to the $2016 \mathrm{WHO}$ Global Health Observatory, the total infant mortality rate in Brazil was approximately 2.5 -fold higher than that in the United States (7). Specifically, the rate of infant mortality due to preterm birth was 1.44-fold higher in Brazil compared with the United States, while the rate of infant death due to congenital anomalies in Brazil was 2.25-fold higher than that in United States (7). Despite these observations, the exact reason for the varying rate of ZIKV-associated fetal abnormalities remains elusive. In order to investigate the association between ZIKV infection and congenital defects, a better understanding of the immune crosstalk at the maternal-fetal interface during infection is required to provide insights into ZIKV pathogenesis and fetal disease outcome.

Pathogenic infections during pregnancy have been associated with a high risk of severe complications in both mother and child (8). Infections during late pregnancy are widely associated with severe disease complications that can lead to mortality $(9,10)$ - a clear demonstration of how pathogens establish unique immune adaptations in the context of pregnancy. In light of the gaps in knowledge, we sought to characterize the immune responses upon ZIKV infection that occurred at different stages of pregnancy and to identify potential biomarkers associated with abnormal fetal outcomes.

\section{Results}

Demographics and clinical characteristics of pregnant women. To elucidate the correlation between ZIKV pathogenesis and maternal immunity, we performed an immune profiling analysis of 74 serum specimens derived from healthy pregnant women (control) in Los Angeles (LA) and Rio de Janeiro, Brazil (RJ), and symptomatic ZIKV ${ }^{+}$pregnant women in different trimesters of pregnancy (Table 1). Serum specimens of $\mathrm{RJ}$ healthy controls $(n=30)$ and $\mathrm{ZIKV}^{+}$pregnant women $(n=30)$ were collected from individuals residing in the ZIKV-endemic region - Rio de Janeiro - from September 2015 to May 2016. In addition, serum specimens of healthy controls (LA healthy controls; $n=14$ ) were obtained from pregnant women residing in a ZIKV-non-endemic region (LA) from June 2016 to May 2017. All ZIKV-infected pregnant women were recruited based on the presence of rash and subsequently confirmed for ZIKV infection by quantitative real-time PCR (qRT-PCR) detection in blood (80\%) and/or urine $(20 \%)$ specimens. The Ct values of ZIKV qRT-PCR ranged 29-35 for the first trimester, 28-37 for the second trimester, and 24-37 for the third trimester. In contrast, RJ healthy controls were recruited based on the lack of clinical findings. Follow-up on birth outcomes was performed for all $\mathrm{ZIKV}^{+}$pregnant women. Among the $\mathrm{ZIKV}^{+}$pregnant women, $50.0 \%$ had normal fetal outcomes, with $6.7 \%$ having preterm birth and $43.3 \%$ term birth. On the other hand, the remaining $50 \%$ of $\mathrm{ZIKV}^{+}$pregnant women gave birth with abnormal outcomes: miscarriages (6.7\%), preterm births (16.7\%), and term births $(26.7 \%)$. Birth abnormalities of babies from $15 \mathrm{ZIKV}^{+}$women included convergent strabismus, eye abnormalities and congenital torticollis, hyperexcitability, hyperreflexia, developmental delay, hyper-/hyposignaling in brain MRIs, hyper-/hypotonia, spasticity, and slow primitive reflexes or hypoactivity, which were diagnosed by physical examination and/or neuroimaging (Table 1).

Differential expression of inflammatory cytokines and chemokines elicited by acute ZIKV infection during pregnancy. To characterize immune responses during acute ZIKV infection, immune profiles in LA healthy control sera and $\mathrm{ZIKV}^{+}$sera were compared, revealing dysregulated expressions of 47 cytokines. Specifically, the levels of 22 inflammatory cytokines and 11 chemokines were significantly induced in $\mathrm{ZIKV}^{+}$serum specimens, whereas those of 5 inflammatory cytokines (IL1B, IL2, IL16, IL26, and IFNG) and 9 chemokines (CCL11, CCL19, CCL3, CCL13, CCL21, CCL7, CXCL8, and CXCL12) were detectably repressed (Figure 1, A and B, and Supplemental Table 1).

Ingenuity pathway analysis (IPA) indicated that a panel of cytokines (IL10, CCL8, CCL15, CXCL9, TSLP, CCL23, CCL1, CCL2, CXCL10, and CX3CL1) induced in ZIKV ${ }^{+}$sera appeared to be associated with the activation of chemotaxis of monocytes and NK cells (Figure 2A). In contrast, the repression of IL16, CCL21, CCL19, CCL20, CXCL12, CCL11, CCL3, CXCL8, and IFNG cytokines in ZIKV+ sera was predicted to associate with the inhibition of chemotaxis of $\mathrm{T}$ and $\mathrm{B}$ cells (Figure 2A). In order to determine the correlation between these 2 panels of cytokines, as well as the association of their expressions with ZIKV burdens, we performed Pearson's $r$ correlation analyses. Interestingly, they showed an inverse correlation between total expressions of chemokines and inflammatory cytokines (Figure 2B), a positive correlation between viral burdens and inflammatory cytokine levels (Figure 2C), and no correlation between 
Table 1. Baseline demographic and clinical characteristics of pregnant women included in the present study

\begin{tabular}{|c|c|c|c|}
\hline Clinical parameters & $\begin{array}{l}\text { LA healthy controls } \\
\qquad(n=14)\end{array}$ & $\begin{array}{l}\text { RJ healthy controls } \\
\qquad(n=30)\end{array}$ & $\begin{array}{c}\text { Symptomatic ZIKV+ patients } \\
\qquad(n=30)\end{array}$ \\
\hline \multicolumn{4}{|l|}{ Stages of pregnancy } \\
\hline First trimester ( 0 to <12 wk) & 5 & 10 & 10 \\
\hline Second trimester (12-28 wk) & 5 & 10 & 10 \\
\hline Third trimester (>28 wk) & 4 & 10 & 10 \\
\hline \multicolumn{4}{|l|}{ Age } \\
\hline First trimester ( 0 to $<12$ weeks) & Negative & Negative & 29-35 \\
\hline Second trimester (12-28 wk) & Negative & Negative & $28-37$ \\
\hline Third trimester (>28 wk) & Negative & Negative & $24-37$ \\
\hline \multicolumn{4}{|l|}{ Symptoms, no. (\% total) } \\
\hline Rash & $0(0 \%)$ & $0(0 \%)$ & 30 (100.0\%) \\
\hline Dysuria & $0(0 \%)$ & $0(0 \%)$ & $1(3.3 \%)$ \\
\hline Fever & $0(0 \%)$ & $0(0 \%)$ & $9(30.0 \%)$ \\
\hline $\begin{array}{l}\text { Neurologic symptoms: headache, photophobia, } \\
\text { retro-orbital pain, tremor, or paresthesia }\end{array}$ & $0(0 \%)$ & $0(0 \%)$ & $0(0.0 \%)$ \\
\hline \multicolumn{4}{|l|}{ Pregnancy outcomes for ZIKV+ patients, no. (\% total) } \\
\hline Normal birth - term birth, no abnormalities & N/A & N/A & $15(50.0 \%)$ \\
\hline Preterm birth & N/A & N/A & $2(6.7 \%)$ \\
\hline Term birth & N/A & N/A & $13(43.3 \%)$ \\
\hline Abnormal birth: confirmed and potential & N/A & N/A & $15(50.0 \%)$ \\
\hline Miscarriage & N/A & N/A & $2(6.7 \%)$ \\
\hline Preterm birth & N/A & N/A & $5(16.7 \%)$ \\
\hline
\end{tabular}

Convergent strabismus, hyperexcitability, hyperreflexia, developmental delay, hyper/hyposignaling, hyper/hypotonia, eye abnormalities, spasticity, congenital torticollis, slow primitive reflexes, hypoactivity

viral burdens and chemokine levels (Figure 2D). These results collectively indicate that ZIKV infection during pregnancy induces a large panel of inflammatory cytokines but a limited subset of chemokines. Interestingly, none of the key proinflammatory cytokines, such as IL6, IL1B, IFNG, and TNF, were highly induced, suggesting that ZIKV infection may trigger unique immunoregulatory effects that prevent overt inflammation during pregnancy.

Immune mediators involved in symptomatic onset of ZIKV $V^{+}$pregnancy. During the 2015-2016 ZIKV outbreak in Brazil, arboviruses including dengue virus (DENV) and chikungunya virus (CHIKV) co-circulated in Rio de Janeiro (11). Despite the absence of acute infection in the LA healthy and RJ healthy controls, comparison of these 2 control groups revealed a significant increase of 29 inflammatory cytokines and 8 chemokines in the RJ healthy controls (Supplemental Table 2). This suggests that RJ healthy controls might have had pre-exposure to other co-circulating viruses. To identify cytokines specifically associated with symptomatic ZIKV infection during pregnancy, we compared the immunoprofiles between 30 symptomatic $\mathrm{ZIKV}^{+}$ patients and 30 RJ healthy controls. Twenty-four cytokines (e.g., CXCL10, TNFRSF8, CCL8, CCL2, and CXCL11) were induced and 21 cytokines (e.g., MIF, IL8, CXCL12, CXCL13, CCL20, and CCL7) were repressed in symptomatic $\mathrm{ZIKV}^{+}$patients as compared with RJ healthy controls (Figure 3, A and B).

IPA network analysis identified the potential associations of those 45 cytokines with ZIKV-induced clinical symptoms, including fever, eye inflammation (conjunctivitis), respiratory system (cough or sore throat), skin (rash), joint (arthralgia or arthritis), urinary tract inflammation (dysuria), and neuroinflammation (Figure 3C). Specifically, this network analysis highlighted the potential association of 6 cytokines 
A

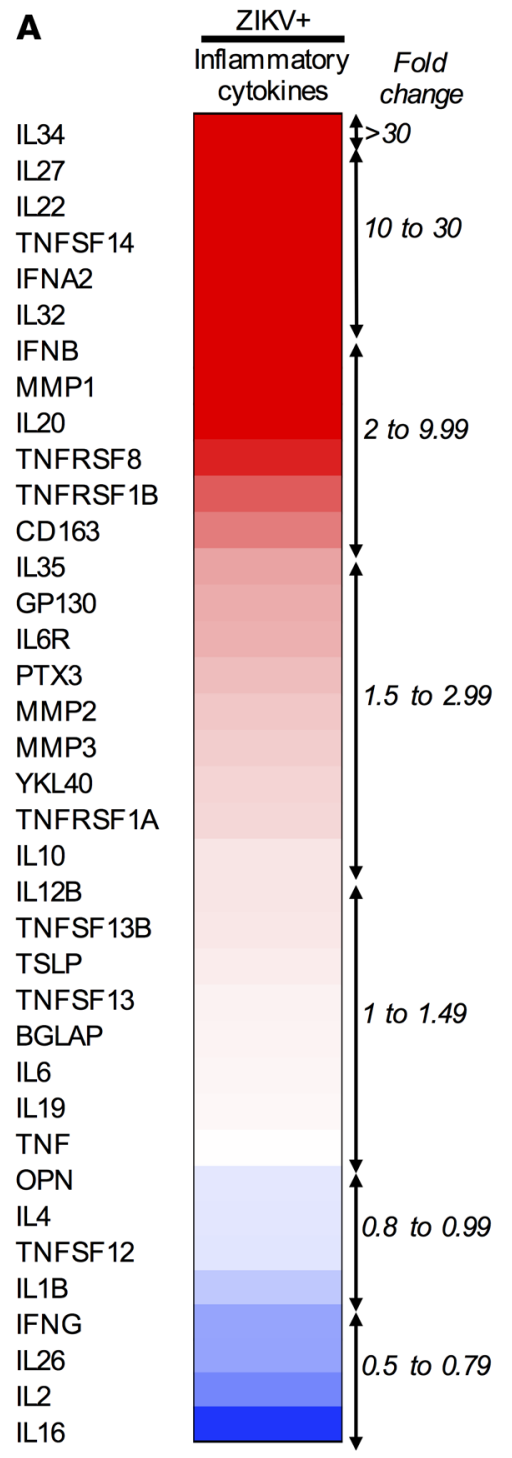

IL16

No change (1-fold)

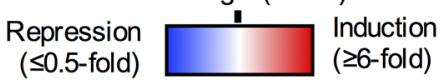

Fold change relative to LA healthy controls
B

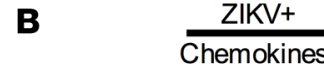

CXCL10

$\mathrm{CCL} 2$

CCL8

CXCL11

CCL15

CX3CL1

$\mathrm{CCL} 24$

CXCL9

CXCL5

CCL23

CXCL2

CCL1

CCL20

CXCL16

CCL26

CXCL6

CXCL1

MIF

CCL22

CCL25

CCL17

GMCSF

CCL27

CCL11

CCL19

CXCL13

$\mathrm{CCL3}$

CCL13

CCL21

CCL7

CXCL8

CXCL12

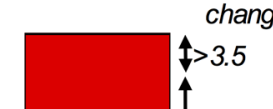

Fold

change

2 to 3.5

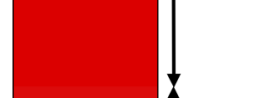

1.2 to 1.99

Figure 1. Differential sera immunoprofiles of pregnant women with acute ZIKV infection. Multiplex immune profiling of 37 inflammatory cytokines and 32 chemokines using serum specimens derived from 14 LA healthy pregnant women ( $n=4-5 /$ trimester) and 30 symptomatic ZIKV+ women ( $n=10 /$ trimester). Heatmaps of (A) 37 inflammatory cytokines and (B) 32 chemokines are listed, with fold changes relative to LA healthy controls. Red, white, and blue indicate induction, no change, and repression, respectively.
1 to 1.19

0.7 to

0.99

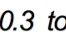

0.69

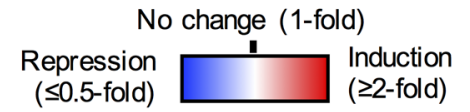

Fold change relative to LA healthy controls

(GP130, IFNB, TNFRSF1A/B, IL22, and IL27 p28) with eliciting symptoms such as rash, respiratory symptoms, conjunctivitis, arthralgia/arthritis, and neurologic symptoms (Figure 3C and Supplemental Figure $1 \mathrm{~A}$ ). On the other hand, the network analysis also highlighted that the 13 repressed cytokines (MIF, CXCL8, CXCL12, CCL7, TNFSF12, CCL3, IL1ß, IL2, OPN, IFNB, IL16, GMCSF, and CCL11) might be associated with the inhibition of ZIKV infection-induced symptoms, particularly fever (Figure 2C and Supplemental Figure 1B). Thus, similar numbers of cytokines were induced or repressed at the time of symptom onset during acute ZIKV infection, which might have resulted in the mild clinical symptoms of ZIKV-infected pregnant patients.

Distinct ZIKV-associated immune signatures during different trimesters of pregnancy. The immune system of a pregnant woman is heavily shaped by the progression of pregnancy (12). In fact, we have previously reported that ZIKV infection of blood in the first and second trimesters of pregnancy enhances viral replication and immune suppression (13). To identify immune signatures associated with ZIKV infection at different trimesters, we performed a trimester-specific comparison of serum immunoprofiles between pregnant RJ healthy and $\mathrm{ZIKV}^{+}$women. In each trimester, we observed a unique cytokine profile: increased CCL23 and decreased 
A

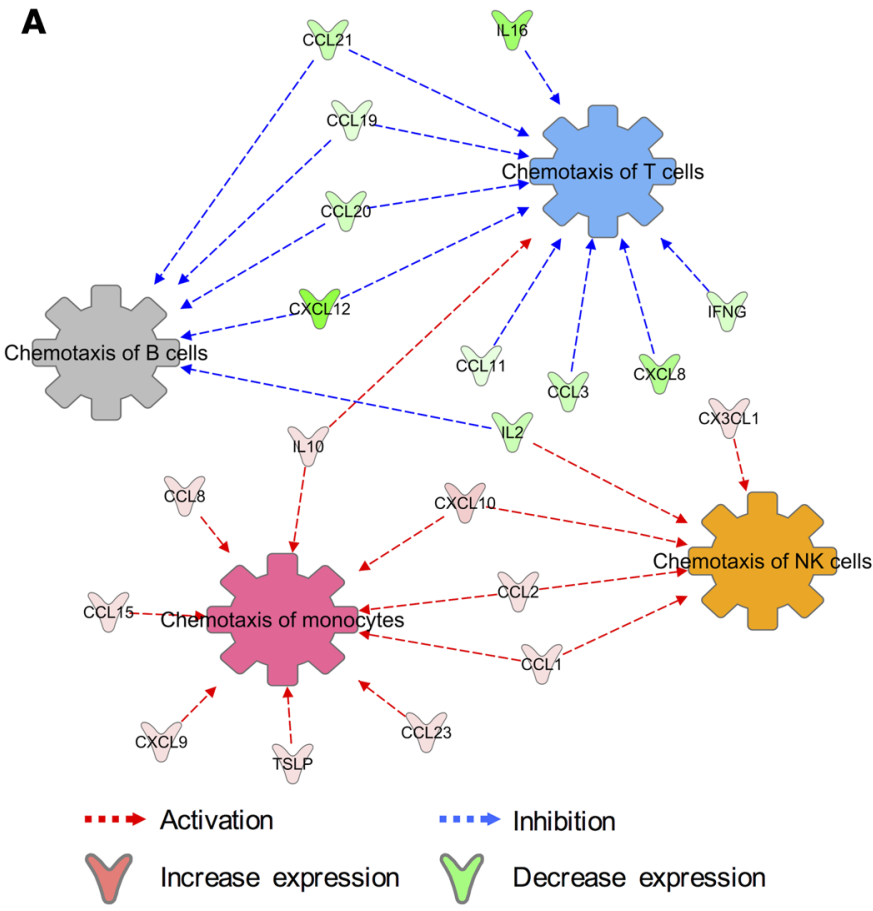

B

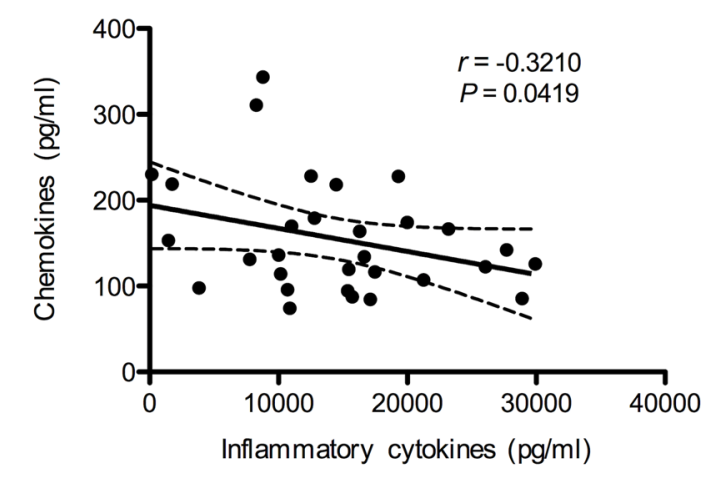

C

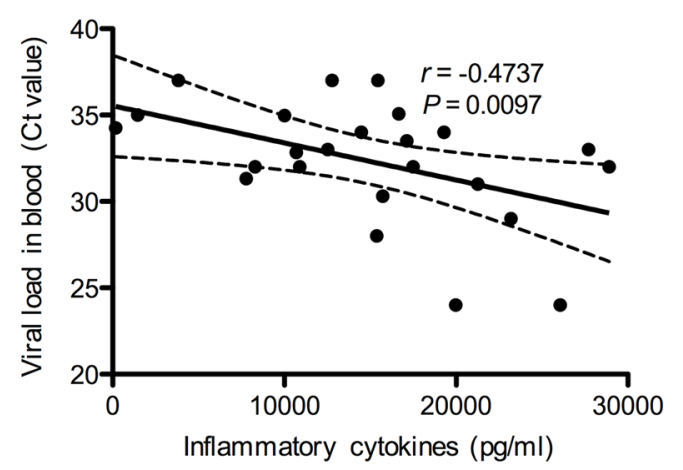

D

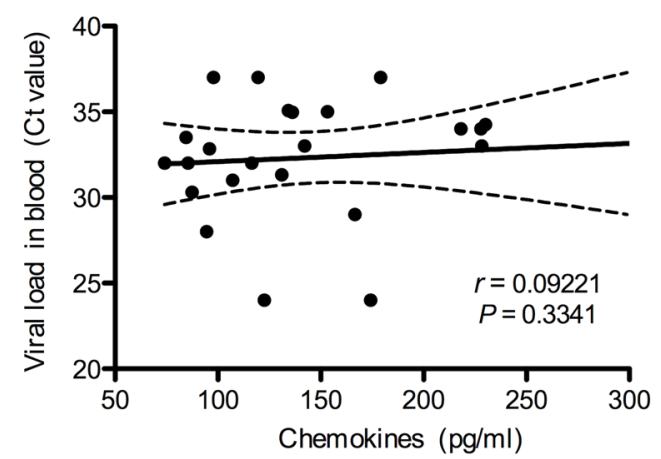

Figure 2. Network analysis of sera immunoprofiles predicted chemotaxis of monocytes and NK cells during ZIKV+ pregnancies. Multiplex immune profiling of 37 inflammatory cytokines and 32 chemokines using serum specimens derived from 14 LA healthy pregnant women $(n=4-5 /$ trimester) and 30 symptomatic ZIKV+ women ( $n=10 /$ trimester). (A) IPA analysis of proteins predicts the chemotaxis of major PBMC subsets: monocytes, NK cells, T cells, and B cells. (B) Correlation analysis between the total expression of inflammatory cytokines and chemokines. Viral load in ZIKV+ pregnant women and total expression of (C) inflammatory cytokines and (D) chemokines $(n=24)$. Correlation analyses were performed using Pearson's $r$ test. Solid lines, linear regression lines; dashed lines, 95\% confidence interval.

TNFSF12 in the first trimester; increased CXCL6, TNFRSF1A, PTX3, and IFNA2 and decreased IL12B in the second trimester; and increased IL22, MMP1, IL20, IL27 p28, and CCL1 and decreased CCL13, GMCSF, CXCL5, and IL19 in the third trimester (Figure 4A). In addition, 10 cytokines were commonly affected by ZIKV infection in all 3 trimesters of pregnancy: CCL8, TNFRSF8, TNFRSF1B, TNFSF14, and CX3CL1 were significantly increased, whereas CCL20, IFNG, CCL7, CXCL13, and MIF were decreased (Figure 4B).

As RJ healthy controls were likely to have a primed immunity against co-circulating arboviruses in Brazil, we also compared the immunoprofiles of $\mathrm{ZIKV}^{+}$women and LA healthy controls at 3 different trimesters (Supplemental Figure 2A). This revealed different immune signatures of $\mathrm{ZIKV}^{+}$women upon comparison with RJ healthy controls or LA healthy controls. However, the increase in CCL23 in the first trimester, the increase in CCL1 in the third trimester, the increase in CCL15 and CCL2 in the second and third trimesters, and the decrease in CCL13 in the third trimester were commonly observed in ZIKV+ women when compared with both RJ healthy controls and LA healthy controls. Also, the increase in TNFRSF1B and CX3CL1 and 
A

CXCL10
TNFRSF8
CCL8
CCL2
CXCL11
IFNA2
TNFSF14
TNF-R2
CD163
CCL15
CXCL9
IL27
IL6R
IFNB
gp130
CCL23
CX3CL1
PTX3
TNFSF13B
MMP1
IL22
IL20
TNFRSF1A
CCL1
CCL11
CXCL6
GMCSF
IL16
CCL13
CCL19
IFNG
OPN
BGLAP
IL2
IL1B
CCL3
BGLAP
TNFSF12
IL26
CCL7
CCL20
CXCL13
CXCL12
IL8
MIF

MIF

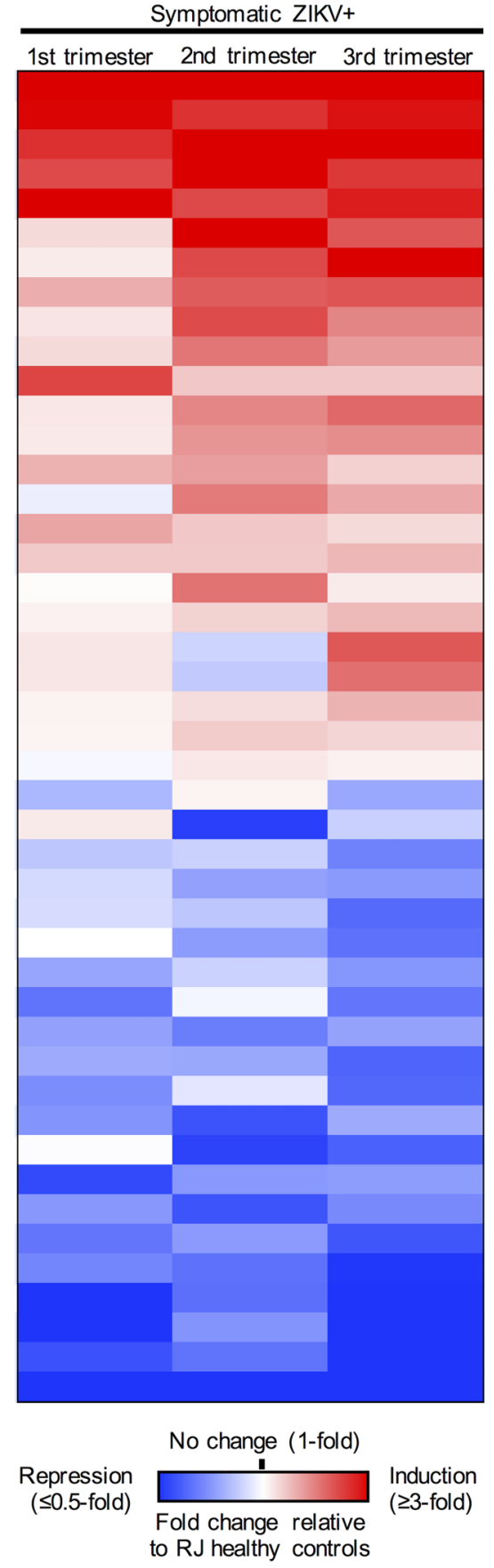

B
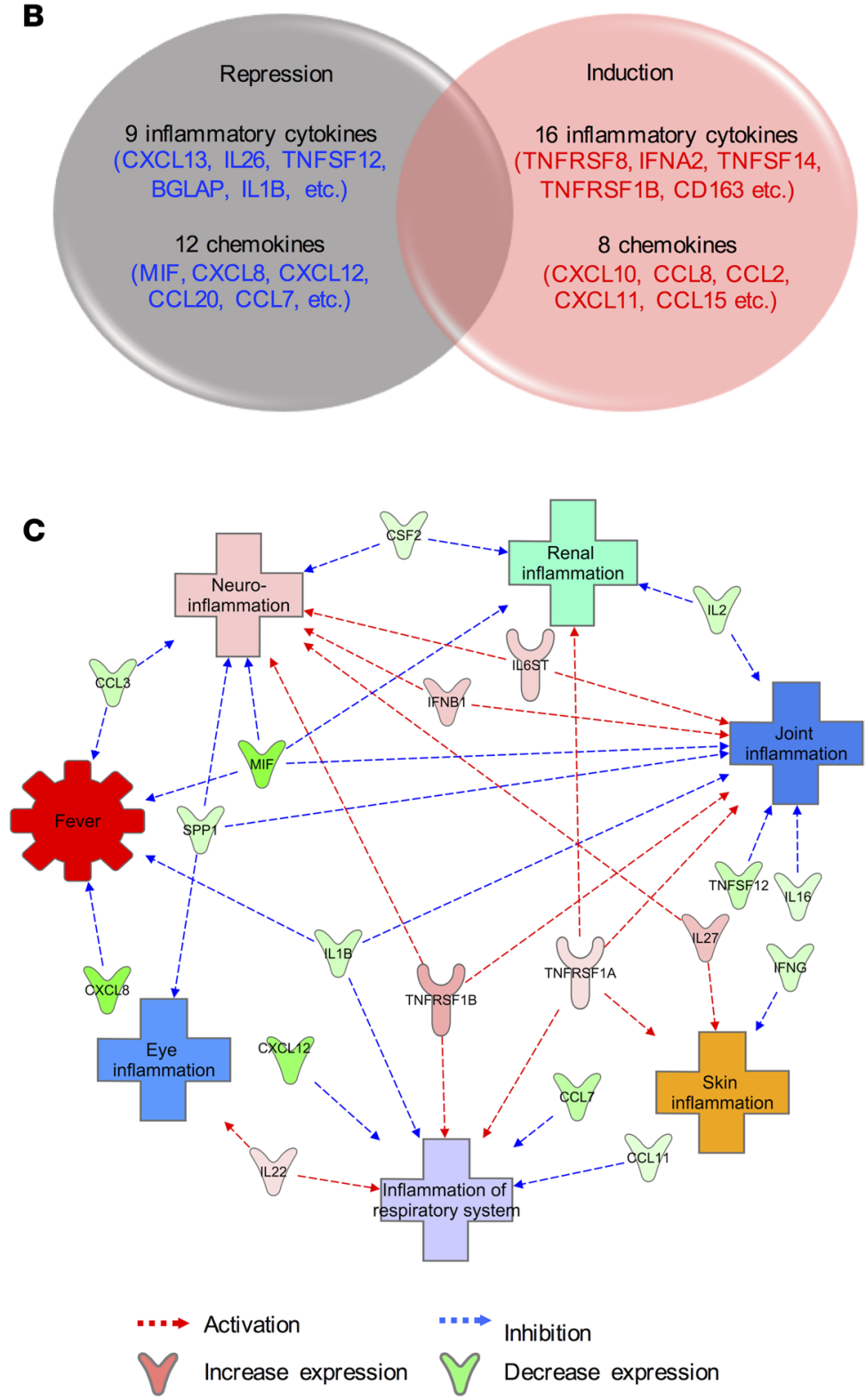

Figure 3. Immune mediators associated with symptomatic onset in ZIKV+ pregnant women from Rio de Janeiro. Multiplex immune profiling of 45 inflammatory cytokines and chemokines using serum specimens derived from 30 healthy pregnant women ( $n=10 /$ trimester) and 30 symptomatic ZIKV ${ }^{+}$women ( $n=10 /$ trimester) from Rio de Janeiro, Brazil (RJ). (A) Heatmap of 45 inflammatory cytokines and chemokines that were significantly increased/decreased when compared with RJ healthy controls. Fold changes in cytokines are relative to RJ healthy controls and presented as heatmap. Red, white, and blue indicate induction, no change, and repression, respectively. (B) Venn diagram representation of number of inflammatory cytokines and chemokines significantly induced/repressed during symptomatic ZIKV+ pregnancy. (C) IPA analysis of proteins predicts the connection of cytokines with various ZIKV-associated clinical manifestations.

the decrease in CCL7 were commonly observed in all 3 trimesters (Supplemental Figure 2B). These comparative analyses demonstrate potential trimester-specific immune signatures upon ZIKV infection.

A unique subset of immune signatures is associated with ZIKV-induced fetal abnormalities. In order to explore the correlation between altered maternal immunity and fetal abnormality of $\mathrm{ZIKV}^{+}$patients, we attempted to identify potential immune signatures that might be useful in predicting fetal abnormalities. Fetal outcomes 
A

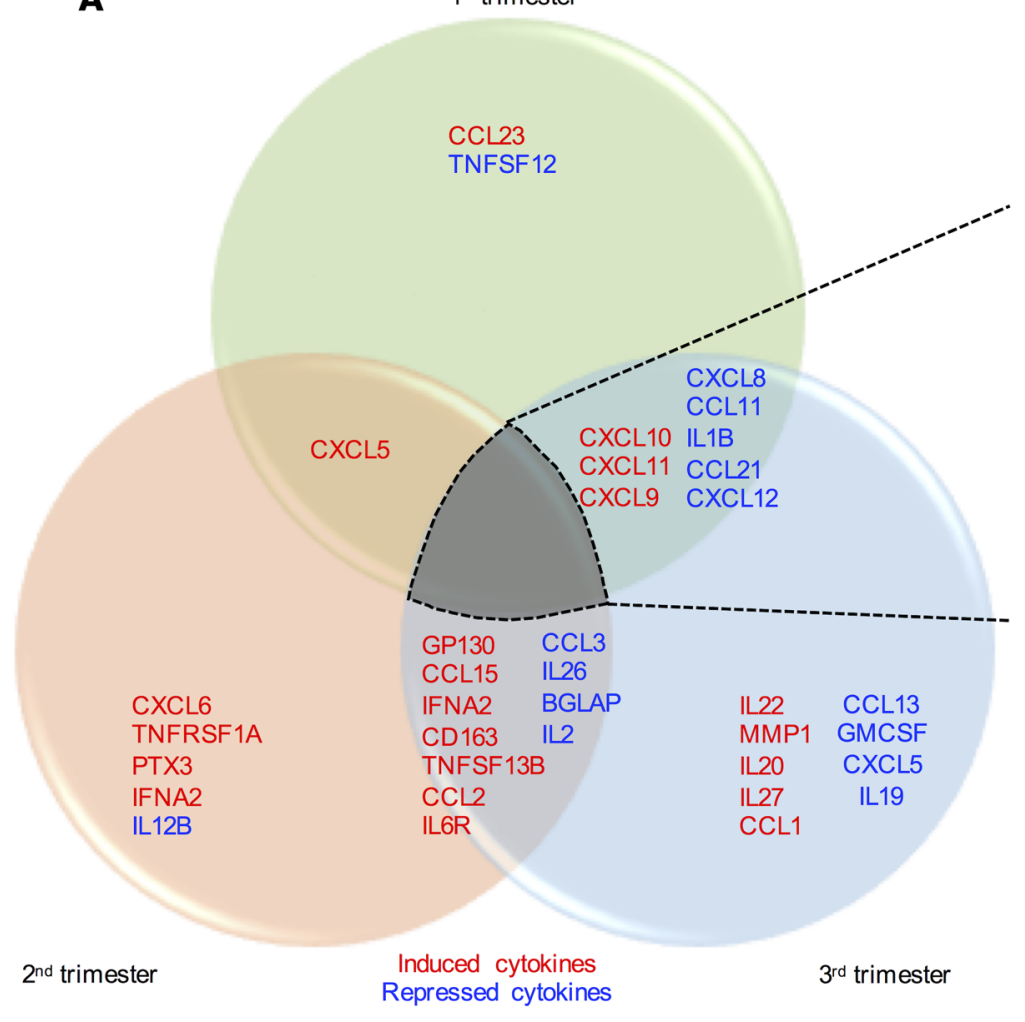

B

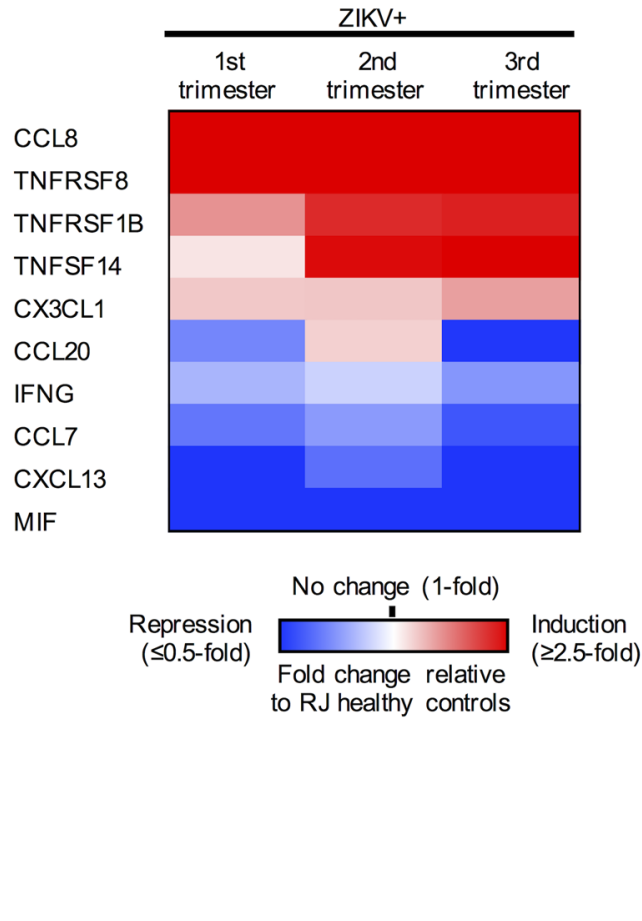

Figure 4. Distinct immune signatures of symptomatic ZIKV infection in different trimesters of pregnancy. Multiplex immune profiling of 30 inflammatory cytokines and 39 chemokines using serum specimens derived from $30 \mathrm{RJ}$ healthy pregnant women ( $n=10 /$ trimester) and 30 symptomatic ZIKV+ women ( $n=10$ /trimester). (A) Venn diagram representation of distinct immune signatures of acute ZIKV infection in each trimester. Red, induced cytokines; blue, repressed cytokines. (B) Heatmap of 10 inflammatory cytokines/chemokines. Fold changes in cytokines are relative to Rio de Janeiro (RJ) healthy and presented as heatmap. Red, white, and blue indicate induction, no change, and repression, respectively.

were determined in the $\mathrm{ZIKV}^{+}$patient cohort at the time of birth (Table 1). $\mathrm{ZIKV}^{+}$pregnant women were subcategorized into normal $(n=15)$ and abnormal $(n=15)$ birth outcomes. We performed statistical analyses between these 2 groups to determine the cytokines associated with ZIKV-induced fetal abnormalities. This identified 16 cytokines that were significantly induced (Figure 5A). IPA analysis of these 16 cytokines predicted their association with promoting inflammation, monocyte and lymphocyte transmigration, and tissue fibrosis (Figure 5B). Particularly, CCL2 was found to be the most highly induced cytokine in $\mathrm{ZIKV}^{+}$pregnant women with abnormal birth outcomes relative to $\mathrm{ZIKV}^{+}$pregnant women with normal birth outcomes (Figure $5 \mathrm{~A}$ and Supplemental Figure $3 \mathrm{~A}$ ). To identify additional biomarker(s) associated with $\mathrm{ZIKV}^{+}$pregnant women with fetal abnormalities, we performed Pearson's $r$ correlation analyses to compare CCL2 with the remaining 15 cytokines upregulated in $\mathrm{ZIKV}^{+}$pregnant women with abnormal fetal outcomes (Supplemental Figure 3B). Among these 15 cytokines, CCL8 and CXCL9 exhibited a positive correlation with CCL2 in pregnant women with abnormal fetal outcomes (Figure 5C). Interestingly, CD163, TNFRSF1A, and CCL22 demonstrated a negative correlation with CCL2 in pregnant women with abnormal fetal outcomes. However, there was a positive or no correlation with CCL2 in pregnant women with normal fetal outcomes (Figure 5C). Finally, we identified significantly increased CCL2/CD163, CCL2/TNFRSF1A, and CCL2/CCL22 ratios in $\mathrm{ZIKV}^{+}$pregnant women with fetal abnormalities, when compared with LA/RJ healthy control or $\mathrm{ZIKV}^{+}$ pregnant women with normal fetal outcomes (Figure 5D). These augmented ratios were further confirmed by ELISAs (Supplemental Figure 4). No statistically significant changes were observed in the CCL2/CCL8 and CCL2/CXCL9 ratios across healthy control and $\mathrm{ZIKV}^{+}$pregnant women with or without fetal abnormalities (Supplemental Figure 3C). Hence, our findings identified 16 cytokines and 3 unique cytokine ratios that were considerably increased in pregnant women with ZIKV-associated fetal abnormalities, suggesting that they may serve as potential immune signatures for predicting birth abnormalities in $\mathrm{ZIKV}^{+}$pregnant women. 
A

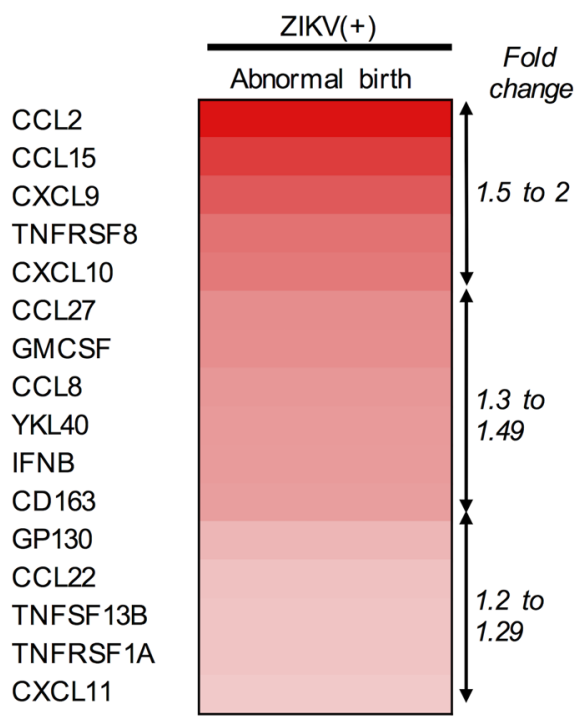

$(\geq 1$-fold $)$

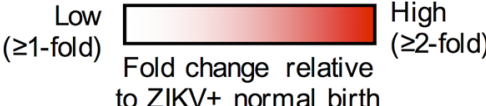

D

CCL2:SCD163

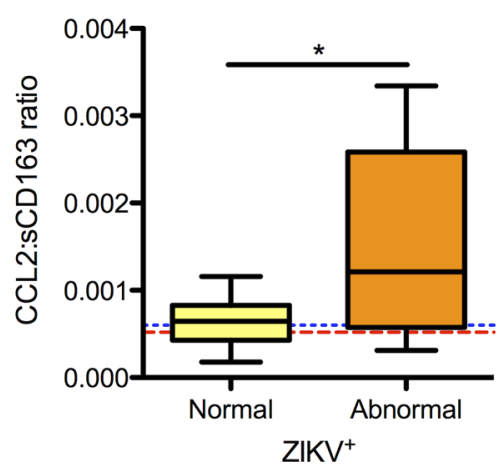

B

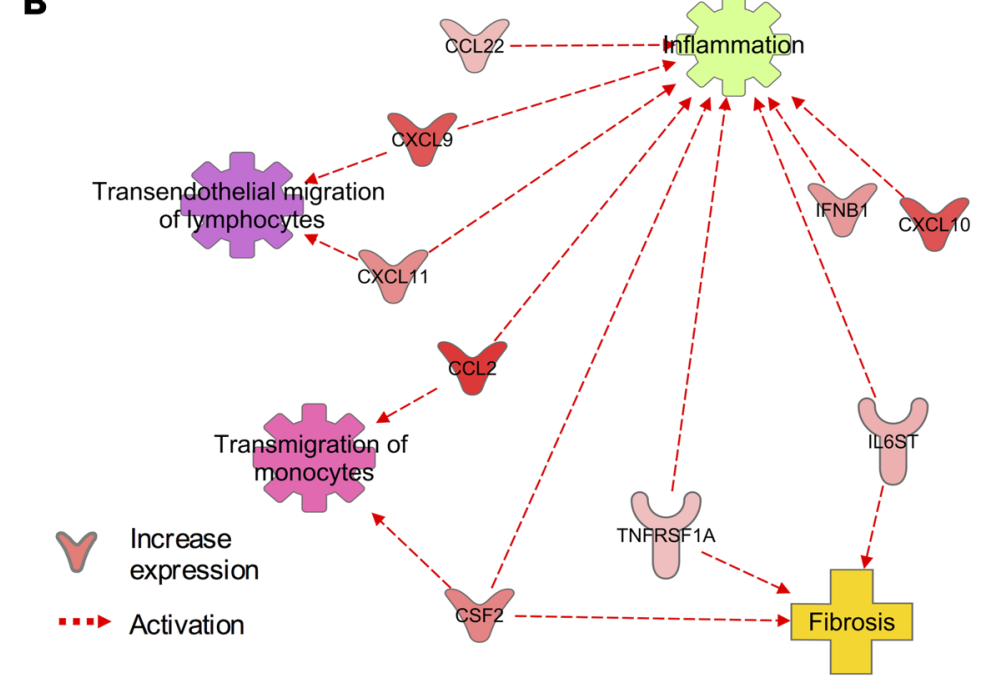

C

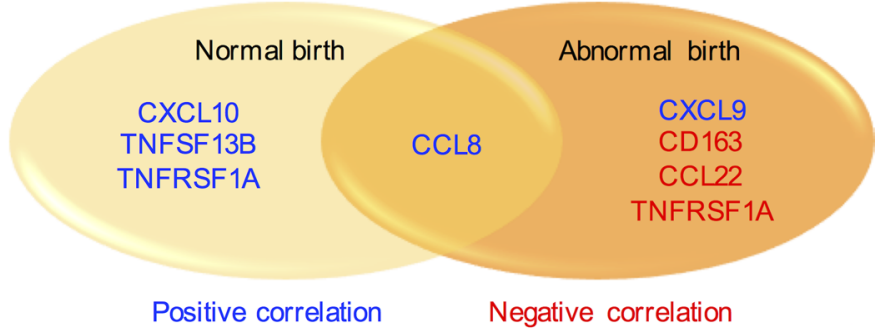

CCL2:TNFRSF1A

CCL2:CCL22

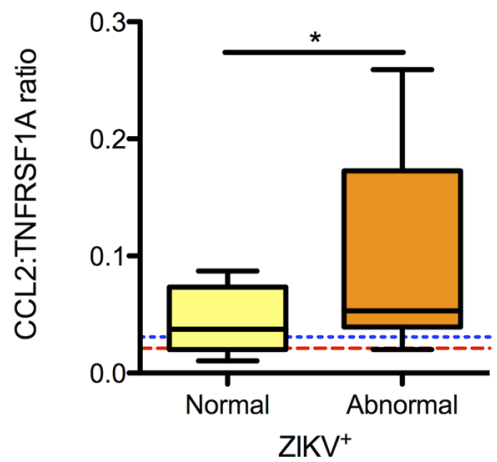

Figure 5. Biomarkers associated with abnormal fetal outcomes. Multiplex immune profiling of 69 inflammatory cytokines and chemokines using serum specimens derived from $14 \mathrm{LA}$ healthy pregnant women $\left(n=4-5 /\right.$ trimester), $30 \mathrm{RJ}$ healthy pregnant women $\left(n=10 /\right.$ trimester), and $30 \mathrm{ZIKV}{ }^{+}$ pregnant women ( $n=10$ /trimester). (A) Heatmap and fold change of 16 inflammatory cytokines significantly upregulated in ZIKV+ women with abnormal birth compared with ZIKV+ women with normal birth. (B) IPA analysis of induced cytokines predicts cellular events associated with maternal-fetal cellular trafficking, inflammation, and tissue fibrosis. (C) Venn diagram representation of cytokines negatively/positively correlated to CCL2 expression in ZIKV+ pregnancy with normal or abnormal birth outcomes. (D) Increased CCL2/CD163, CCL2/TNFRSF1A, and CCL2/CCL22 ratios in ZIKV+ pregnancy with abnormal birth outcomes. Data (mean \pm SEM) are presented in the box plot showing upper $(75 \%)$ and lower $(25 \%)$ quartiles, with horizontal line as median and whiskers as maximum and minimum values. ${ }^{*} P<0.05$, Mann-Whitney $U$ test. Median value of the ratios for LA healthy and $R J$ healthy controls is represented as blue dotted line and red dotted line, respectively.

\section{Discussion}

While $80 \%$ of ZIKV-infected adult individuals may not exhibit any symptoms, $20 \%$ of the patients develop mild symptoms, including rash, arthralgia, conjunctivitis, and low-grade fever, which rapidly resolve within a week after onset of illness (14). Other less common clinical symptoms include dysuria and neurological symptoms such as photophobia, retro-orbital pain, tremor, and paresthesia, whereas Guillain-Barré syndrome can be seen in more severe ZIKV cases (15). In this study, we sought to 
characterize the association between immune responses and clinical outcomes during acute ZIKV infection of pregnant women through extensive immunoprofiling analyses.

Among the 69 cytokines screened, we identified a subset of 45 cytokines dysregulated during acute infection by comparing the immunoprofiles of symptomatic $\mathrm{ZIKV}^{+}$pregnant women and healthy pregnant women in Rio de Janeiro. Intriguingly, 24 of 45 dysregulated cytokines were upregulated, and CXCL10 (median of $411.4 \mathrm{pg} / \mathrm{ml}$ ) was the most highly induced cytokine in symptomatic $\mathrm{ZIKV}^{+}$pregnant women. Indeed, this CXCL10 level in ZIKV patients is on par with that of patients with dengue hemorrhagic fever (approximately $500 \mathrm{pg} / \mathrm{ml}$ ), who exhibit considerable morbidity with high-grade fever and debilitating arthralgia $(16,17)$. As previously described (18-21), immunosuppressive cytokines including IL34, IL27, and IL35 were also strongly induced in ZIKV+ pregnant women. We have previously demonstrated that the infection of blood in pregnant women with epidemic ZIKV strain leads to the overt M2 macrophage-skewed immunosuppression through the apparent expression of immunosuppressive IL10, CD163, and YKL40 molecules (13). These suggest that antiinflammatory cytokines (IL10, CD163, YKL14, IL34, IL27, and IL35) may counteract proinflammatory cytokine CXCL10, ultimately resulting in the mild onset of symptoms in pregnant women with acute ZIKV infection. In addition, pathway analysis also showed that a majority of the cytokines induced by ZIKV infection were involved in the recruitment of monocytes and NK cells. In fact, the excessive monocyte and NK cell infiltration to the maternal-fetal interface has been linked to pregnancy complications such as preeclampsia and preterm birth $(22,23)$. Thus, the ZIKV-induced cytokine milieu during pregnancy may result in infiltration of leukocyte, particularly monocytes and NK cells, which ultimately contributes to viral pathogenesis.

The cytokine profiling of $\mathrm{ZIKV}^{+}$pregnant women suggests that infection-induced virulence can be shaped by pregnancy immunity. In this study, we identified unique subsets of cytokines that were dysregulated at specific trimesters of pregnancy upon ZIKV infection. Due to the short viremic phase of ZIKV, many infected pregnant women might have been identified to be negative for ZIKV infection by qRT-PCR. Furthermore, the serodiagnosis of ZIKV remains challenging due the high cross-reactivity with DENV (24, 25). The identification of this specific panel of biomarkers elicited at different stages of pregnancy can be a useful tool to identify pregnant women who may have suspected ZIKV infection and/or may be at risk of developing ZIKV-associated fetal abnormalities.

Growing evidence has suggested the negative impact of maternal inflammation on fetal neurodevelopment (26), suggesting that ZIKV-mediated immunomodulatory strategy during pregnancy may be the underlying cause of abnormal birth outcomes. However, the studies of potential biomarkers to predict ZIKV-associated fetal abnormalities are limited to date. A recent report by Kam et al. has suggested IL18 and CXCL10 as possible biomarkers for ZIKV-associated fetal abnormalities of a single ZIKV-infected pregnant woman whose fetus had congenital anomalies (27). We compared $15 \mathrm{ZIKV}^{+}$ pregnant women with healthy baby births and $15 \mathrm{ZIKV}^{+}$pregnant women with abnormal fetal outcomes and observed increased expression of 16 inflammatory cytokines. Among these 16 inflammatory cytokines, CCL2 not only was induced at the highest level but also showed a unique inverse relationship with CD163, TNFRSF1A, and CCL22 in ZIKV ${ }^{+}$pregnant women with abnormal birth outcomes. This suggest that the CCL2 level and its inverse relationship with CD163, TNFRSF1A, and CCL22 may serve as potential biomarkers to predict ZIKV-associated fetal abnormalities. As in other pregnancy disorders such as preeclampsia, it is unlikely that a single biomarker can be an accurate predictive tool for ZIKV fetal abnormalities. Therefore, our extensive immunoprofiling study of the prospective $\mathrm{ZIKV}^{+}$pregnant cohort with known fetal outcomes has identified several biomarkers that may play indispensable roles in the diagnosis of ZIKV-associated fetal abnormalities. However, the limitations of this study include (i) the inability to correlate these findings to asymptomatic $\mathrm{ZIKV}^{+}$pregnant subjects, since symptomatic onset was the sole inclusion criteria for subsequent ZIKV diagnosis and follow-up, and (ii) the inability to obtain specimens from pregnant subjects with "clean" infection history in Rio de Janeiro due to the co-circulation of multiple arboviruses before and during the time of ZIKV outbreak. Future studies are required not only to further validate these specific biomarkers associated with morbidity, but also to improve the clinical management of long-term ZIKV-associated abnormalities in affected infants. 


\section{Methods}

Human subjects and blood collection. Peripheral blood specimens were obtained from pregnant women aged 16-46 years old: (i) healthy pregnant controls in non-ZIKV endemic Los Angeles and (ii) healthy pregnant controls, as well as (iii) symptomatic ZIKV+ subjects in ZIKV-endemic Rio de Janeiro. Pregnancy was categorized into three trimesters based on the following: first trimester $\leq 13$ weeks, second trimester 14-28 weeks and third trimester $\geq 29$ weeks. Healthy pregnant subjects were recruited at Los Angeles County + University of Southern California (LAC + USC) Medical Center, with the following exclusion criteria: (i) vaccination within last 14 days, (ii) suspected/recent illness within last 14 days, (iii) steroid administration during pregnancy, (iv) immunodeficiency/immunocompromised conditions, (v) autoimmune disorders, and (vi) suspected fetal anomalies on transfontanelle ultrasonography. Blood sera were collected from 30 symptomatic $\mathrm{ZIKV}^{+}$pregnant women, 10 at each trimester, who were admitted to the acute febrile illness clinic at FIOCRUZ, while exhibiting rash that had developed within the previous 5 days. All patients were confirmed to be $\mathrm{ZIKV}^{+}$following the performance of qRT-PCR assay in blood $(80 \%)$ or urine $(20 \%)$ that utilized specific probes and primers for ZIKV detection (24). In addition, 30 healthy pregnant women who did not exhibit any sign of illness were also recruited in this study. All pregnant subjects recruited in this study were negative for DENV, CHIKV, parvovirus B19, cytomegalovirus, and human immunodeficiency virus by PCR assays. Serum aliquots from these patients were stored at $-80^{\circ} \mathrm{C}$ until the performance of the present experiments.

Clinical assessments of all ZIKV-exposed infants were performed by pediatric specialists and further evaluated by a multidisciplinary team comprising: (i) neonatologists, (ii) neurologists, (iii) infectious disease specialists, (iv) geneticists, (v) ophthalmologists, and (vi) physical therapists. Confirmation of abnormal fetal outcomes was made upon final review by a panel of multidisciplinary specialists. No follow-up study was performed on healthy controls from Rio de Janerio and healthy controls from Los Angeles.

Viral load detection assay. Viral load detection of ZIKV in sera and urine specimens was performed using qRTPCR assays with the QuantiTect Probe RT-PCR kit (catalog 204443; QIAGEN), as previously described (24).

Multiplex immunoassay and ELISA. Bio-Plex Pro human inflammation (37-plex; catalog 171AL001M) and chemokine (40-plex; 171AK99MR2) panel assays (Bio-Rad) and CCL2 (438804; BioLegend), TNFRSF1A (DRT100; R\&D Systems), CD163 (DY1607-05; R\&D Systems), and CCL22 ELISAs (DY336; R\&D Systems) were performed on serum specimens according to the manufacturers' instructions. IPA (QIAGEN) was used to predict the associations of cytokines to biological pathways involved in disease and cellular signaling.

Statistics. All statistical analyses were performed using GraphPad Prism 5.0 software. For analyses between 2 groups, Mann-Whitney $U$ test was used. For multiple comparison across 3 groups, 1 -way ANOVA, Bonferroni's post hoc test was used. $P$ values less than 0.05 were considered significant, and are indicated in the figures as follows: ${ }^{*} P<0.05,{ }^{* *} P<0.01$, and ${ }^{* *} P<0.001$. For correlation analyses, Pearson $\mathrm{R}$ tests were performed, with $r$ values $>0.3$ or $<-0.3$ considered positively or negatively correlated, respectively. Linear regression lines and 95\% confidence bands of the best-fit line are shown for all graphs of correlation analyses.

Study approval. All human studies were approved by participating institutional review boards. Protocols were approved by the Institutional Review Board (IRB) of the USC, FIOCRUZ, and UCLA. Details of the recruitment, enrollment, and follow-up of pregnant subjects in Rio de Janeiro have been previously published $(6,28)$. Written and oral informed consents were obtained prior to the collection of serum specimens from all pregnant women involved in this study.

\section{Author contributions}

SSF and WC performed experiment and data analyses of the study. YC, WSL, SAL, GC, KNS, and PB contributed to assembly and collection of human specimens used in this study. SSF, WC, and JUJ directed the conception and design of the study, interpretation of the data, and manuscript writing.

\section{Acknowledgments}

This work was partly supported by NIH grants CA200422, CA180779, DE023926, AI073099, AI116585, AI129496, and AI140705, the Hastings Foundation, and the Fletcher Jones Foundation (JUJ); NIH grants AI069120, AI056154, AI078389, and AI28697 (GC); NIH grants AI129534-01 (KNS) and R01AI40718 (JUU, GC, and KNS) and Departamento de Ciência e Tecnologia (DECIT/25000.072811/2016-17) do Ministério da Saúde do Brasil and Coordenação de Aperfeiçoamento de Pessoal de Nivel Superior CAPES/88887.116627/2016-01 (PB). 
Address correspondence to: Suan-Sin Foo, Weiqiang Chen, or Jae Jung, Department of Molecular Microbiology and Immunology, University of Southern California, Keck Medical School, Zilkha Neurogenetic Institute, 1501 San Pablo Street, Los Angeles, California 90033, USA. Phone: 323.442.1713; Email: foos@usc.edu (SSF), weiqchen@usc.edu (WC), jaeujung@med.usc.edu (JUJ).

1. Zanluca C, Melo VC, Mosimann AL, Santos GI, Santos CN, Luz K. First report of autochthonous transmission of Zika virus in Brazil. Mem Inst Oswaldo Cruz. 2015;110(4):569-572.

2. PAHO and WHO. Zika suspected and confirmed cases reported by countries and territories in the Americas Cumulative cases, 2015-2017. PAHO website. https://www.paho.org/hq/dmdocuments/2017/2017-oct-5-phe-ZIKV-cases.pdf. Updated October 5, 2017. Accessed October 11, 2018.

3. CDC. Outcomes of Pregnancies with Laboratory Evidence of Possible Zika Virus Infection, 2015-2018. CDC website. https:// www.cdc.gov/pregnancy/zika/data/pregnancy-outcomes.html. Updated March 27, 2018. Accessed October 11, 2018.

4. Shapiro-Mendoza CK, et al. Pregnancy outcomes after maternal Zika virus infection during pregnancy - U.S. Territories, January 1, 2016-April 25, 2017. MMWR Morb Mortal Wkly Rep. 2017;66(23):615-621.

5. Reynolds MR, et al. Vital signs: update on Zika virus-associated birth defects and evaluation of all u.s. infants with congenital Zika virus exposure — U.S. Zika Pregnancy Registry, 2016. MMWR Morb Mortal Wkly Rep. 2017;66(13):366-373.

6. Brasil P, et al. Zika virus infection in pregnant women in Rio de Janeiro. N Engl J Med. 2016;375(24):2321-2334.

7. Global Strategy for Women's, Children's and Adolescents' Health (2016-2030). World Health Organization; 2017.

8. Kourtis AP, Read JS, Jamieson DJ. Pregnancy and infection. N Engl J Med. 2014;371(11):1077.

9. Siston AM, et al. Pandemic 2009 influenza A(H1N1) virus illness among pregnant women in the United States. JAMA. 2010;303(15):1517-1525.

10. Kaufman B, Gandhi SA, Louie E, Rizzi R, Illei P. Herpes simplex virus hepatitis: case report and review. Clin Infect Dis. 1997;24(3):334-338

11. Brasil P, et al. Zika Virus Outbreak in Rio de Janeiro, Brazil: clinical characterization, epidemiological and virological aspects PLoS Negl Trop Dis. 2016;10(4):e0004636.

12. Racicot K, Kwon JY, Aldo P, Silasi M, Mor G. Understanding the complexity of the immune system during pregnancy. Am $J$ Reprod Immunol. 2014;72(2):107-116.

13. Foo SS, et al. Asian Zika virus strains target CD14+ blood monocytes and induce M2-skewed immunosuppression during pregnancy. Nat Microbiol. 2017;2(11):1558-1570.

14. Duffy MR, et al. Zika virus outbreak on Yap Island, Federated States of Micronesia. N Engl J Med. 2009;360(24):2536-2543.

15. Cao-Lormeau VM, et al. Guillain-Barré syndrome outbreak associated with Zika virus infection in French Polynesia: a case-control study. Lancet. 2016;387(10027):1531-1539.

16. Oliveira RADS, et al. Serum cytokine/chemokine profiles in patients with dengue fever (DF) and dengue hemorrhagic fever (FHD) by using protein array. J Clin Virol. 2017;89:39-45.

17. Herrero LJ, et al. Dengue virus therapeutic intervention strategies based on viral, vector and host factors involved in disease pathogenesis. Pharmacol Ther. 2013;137(2):266-282.

18. Foucher ED, et al. IL-34 induces the differentiation of human monocytes into immunosuppressive macrophages. antagonistic effects of GM-CSF and IFN $\gamma$. PLoS One. 2013;8(2):e56045.

19. Bézie S, et al. IL-34 is a Treg-specific cytokine and mediates transplant tolerance. J Clin Invest. 2015;125(10):3952-3964.

20. Karakhanova S, Bedke T, Enk AH, Mahnke K. IL-27 renders DC immunosuppressive by induction of B7-H1. J Leukoc Biol. 2011;89(6):837-845

21. Banchereau J, Pascual V, O'Garra A. From IL-2 to IL-37: the expanding spectrum of anti-inflammatory cytokines. Nat Immunol. 2012;13(10):925-931.

22. Faas MM, Spaans F, De Vos P. Monocytes and macrophages in pregnancy and pre-eclampsia. Front Immunol. $2014 ; 5: 298$.

23. Gervasi MT, et al. Phenotypic and metabolic characteristics of maternal monocytes and granulocytes in preterm labor with intact membranes. Am J Obstet Gynecol. 2001;185(5):1124-1129.

24. Lanciotti RS, et al. Genetic and serologic properties of Zika virus associated with an epidemic, Yap State, Micronesia, 2007. Emerging Infect Dis. 2008;14(8):1232-1239.

25. Johansson MA, Mier-y-Teran-Romero L, Reefhuis J, Gilboa SM, Hills SL. Zika and the risk of microcephaly. NEngl J Med. 2016;375(1):1-4.

26. Ginsberg Y, Khatib N, Weiner Z, Beloosesky R. Maternal inflammation, fetal brain implications and suggested neuroprotection: a summary of 10 years of research in animal models. Rambam Maimonides Med J. 2017;8(2):e0028.

27. Kam YW, et al. Specific biomarkers associated with neurological complications and congenital central nervous system abnormalities from Zika virus-infected patients in Brazil. J Infect Dis. 2017;216(2):172-181.

28. Halai UA, et al. Maternal Zika virus disease severity, virus load, prior dengue antibodies, and their relationship to birth outcomes. Clin Infect Dis. 2017;65(6):877-883. 